\title{
Do self-management interventions in COPD patients work and which patients benefit most? An individual patient data meta-analysis
}

This article was published in the following Dove Press journal:

International Journal of COPD

31 August 2016

Number of times this article has been viewed

Nini H Jonkman,' Heleen

Westland,' Jaap CA Trappenburg,'

Rolf HH Groenwold, ${ }^{2}$ Erik WMA

Bischoff, ${ }^{3}$ Jean Bourbeau, ${ }^{4}$

Christine E Bucknall, ${ }^{5}$ David

Coultas, ${ }^{6}$ Tanja W Effing, ${ }^{7}$ Michael J

Epton, ${ }^{8}$ Frode Gallefoss, ${ }^{9}$ Judith

Garcia-Aymerich, ${ }^{10-12}$ Suzanne M

Lloyd, ${ }^{13}$ Evelyn M Monninkhof, ${ }^{2}$

Huong Q Nguyen, ${ }^{14}$ Job van der

Palen, ${ }^{15,16}$ Kathryn L Rice, ${ }^{17}$ Maria

Sedeno, ${ }^{4}$ Stephanie JC Taylor, ${ }^{18}$

Thierry Troosters, ${ }^{19}$ Nicholas A

Zwar, ${ }^{20}$ Arno W Hoes, ${ }^{2}$ Marieke J

Schuurmans

'Department of Rehabilitation, Nursing Science and Sports, 'Julius Center for Health Sciences and Primary Care, University Medical Center Utrecht Utrecht, ${ }^{3}$ Department of Primary and Community Utrecht, 'Department of Primary and Community Care, Radboud University Medical Center, Nijmeg the Netherlands; ${ }^{4}$ Respiratory Epidemiology and Clinical Research Unit, Department of Medicine,

McGill University Health Center, McGill University,

Montreal, QC, Canada; ${ }^{5}$ Department of Respirato

Medicine, Glasgow Royal Infirmary, Glasgow, UK

Veterans Administration Portland Health Care

System and Oregon Health \& Science University,

Portland, OR, USA; 'Department of Respiratory

Medicine, Repatriation General Hospital, Adelaide,

SA, Australia; ${ }^{8}$ Canterbury District Health Board,

Respiratory Services, Christchurch Hospital,

Christchurch, New Zealand; 'Department

of Pulmonary Medicine, Sorlandet Hospital,

Kristiansand, Norway; ${ }^{10} \mathrm{Centre}$ for Research in

Environmental Epidemiology CREAL, "Pompeu

Fabra University, ${ }^{12} \mathrm{CIBER}$ Epidemiología y Salud

Pública CIBERESP, Barcelona, Spain; ${ }^{13}$ Robertson

Centre for Biostatistics, University of Glasgow,

Glasgow, UK; ${ }^{14}$ Department of Research and

Evaluation, Kaiser Permanente Southern California,

Pasadena, CA, USA; ${ }^{15}$ Department of Research

Methodology, Measurement and Data Analysis,

University of Twente, ${ }^{16}$ Department of Clinical

Epidemiology, Medisch Spectrum Twente, Enschede,

Epidemiology, Medisch Spectrum Twente, Enschede,

the Netherlands; ${ }^{15}$ Division of Pulmonary, Aller

Veterans Affairs Health Care Service and University

of Minnesota, Minneapolis, MN, USA; ${ }^{18}$ Centre

Primary Care and Public Health, Queen Mary

University of London, London, UK; ${ }^{19}$ Department

of Rehabilitation Sciences, Catholic University of

Leuven, Leuven, Belgium; ${ }^{20} \mathrm{School}$ of Public Health and Community Medicine, UNSW Australia, Sydney, NSW, Australia

Correspondence: Nini H Jonkman

Department of Rehabilitation, Nursing Science \& Sports,

University Medical Center Utrecht, HP WOI.I2I,

Heidelberglaan 100, 3508 GA Utrecht, the Netherlands

$\mathrm{Tel}+3130613244760$

Emailn.jonkman@umcutrecht.n
Background: Self-management interventions are considered effective in patients with COPD, but trials have shown inconsistent results and it is unknown which patients benefit most. This study aimed to summarize the evidence on effectiveness of self-management interventions and identify subgroups of COPD patients who benefit most.

Methods: Randomized trials of self-management interventions between 1985 and 2013 were identified through a systematic literature search. Individual patient data of selected studies were requested from principal investigators and analyzed in an individual patient data meta-analysis using generalized mixed effects models.

Results: Fourteen trials representing 3,282 patients were included. Self-management interventions improved health-related quality of life at 12 months (standardized mean difference $0.08,95 \%$ confidence interval $[\mathrm{CI}] 0.00-0.16$ ) and time to first respiratory-related hospitalization (hazard ratio $0.79,95 \%$ CI $0.66-0.94$ ) and all-cause hospitalization (hazard ratio 0.80 , 95\% CI 0.69-0.90), but had no effect on mortality. Prespecified subgroup analyses showed that interventions were more effective in males (6-month COPD-related hospitalization: interaction $P=0.006)$, patients with severe lung function (6-month all-cause hospitalization: interaction $P=0.016)$, moderate self-efficacy (12-month COPD-related hospitalization: interaction $P=0.036$ ), and high body mass index (6-month COPD-related hospitalization: interaction $P=0.028$ and 6-month mortality: interaction $P=0.026$ ). In none of these subgroups, a consistent effect was shown on all relevant outcomes.

Conclusion: Self-management interventions exert positive effects in patients with COPD on respiratory-related and all-cause hospitalizations and modest effects on 12-month health-related quality of life, supporting the implementation of self-management strategies in clinical practice. Benefits seem similar across the subgroups studied and limiting self-management interventions to specific patient subgroups cannot be recommended.

Keywords: chronic obstructive pulmonary disease, individual patient data meta-analysis, self-management, subgroup analysis

\section{Introduction}

With over 60 million people affected, COPD is a major global health problem leading to substantial morbidity and mortality. ${ }^{1}$ In addition to the disease burden, COPD requires a major shift in patients' daily life as they need to adhere to drug treatment, implement lifestyle changes, monitor signs and symptoms, and apply decision making on early treatment of exacerbations to prevent complications. ${ }^{2}$ Interventions to improve this self-management behavior in COPD patients have been receiving increasing attention and generally involve patient education and teaching skills to patients for monitoring 
their condition, carrying out medical regimens, and changing their health behavior. ${ }^{3} \mathrm{~A}$ recent systematic review found positive effects on a range of outcomes, including healthrelated quality of life (HRQoL), dyspnea, and health care utilization. $^{4}$

Although the evidence favors self-management interventions, there seems to be a large heterogeneity in the effects of these interventions. Findings from five randomized trials, all based on the self-management program Living Well With COPD, ${ }^{5}$ were contradictory and have raised questions about large scale implementation of selfmanagement interventions in COPD patients. The first two trials reported large positive effects on respiratory-related hospitalization $^{5}$ and the combined endpoint of respiratoryrelated hospitalization and emergency department visit, ${ }^{6}$ but these promising findings could not be replicated in subsequent studies in the $\mathrm{UK}^{7}$ and the Netherlands. ${ }^{8}$ The fifth trial even reported higher mortality rates among patients in the self-management group and recruitment was terminated prematurely. ${ }^{9}$

Several researchers have postulated hypotheses in an attempt to explain the different outcomes of these five trials. The diversity among interventions, study populations, follow-up time, and outcome measures across these five trials compromise a generalization to real life. Patient factors might matter more than assumed to date and it has been suggested that adherence to and uptake of self-management interventions are better in specific subgroups of patients. ${ }^{10}$ Currently, however, evidence on which subgroups are more likely to benefit from or respond negatively to self-management interventions is lacking. With this knowledge, clinicians might be able to target self-management interventions at those patients who benefit most.

Identification of such patient subgroups in individual trials is complicated, as these usually lack power. A metaanalysis of individual patient data (IPD) enables a more reliable subgroup analysis with sufficient power due to the large numbers of patients included and by allowing a similar definition of subgroups across studies. ${ }^{11}$ Collecting the IPD from different trials also enables standardized statistical analyses and inclusion of data on available but unreported endpoints, which has additional advantages for analyzing the main effects of self-management interventions.

The present IPD meta-analysis aims to summarize the evidence on the effectiveness of COPD self-management interventions on relevant outcomes, including HRQoL, hospitalization, and mortality, with a particular focus on identifying subgroups of patients with COPD who are most likely to benefit from self-management interventions.

\section{Methods}

This IPD meta-analysis was conducted according to the guidelines in the Cochrane Handbook for Systematic Reviews of Interventions ${ }^{12}$ and followed a prespecified protocol. ${ }^{13}$

\section{Search and selection of studies}

We searched the electronic databases PubMed, EMBASE, Cochrane Central Register of Controlled Trials, PsycINFO, and CINAHL from January 1985 through June 2013 and scrutinized the reference lists of identified relevant systematic reviews.

With no general agreement on an operational definition of self-management interventions, an international group of self-management research experts set out to reach consensus on the criteria for defining self-management intervention. There is general agreement on the multifaceted nature of selfmanagement interventions. ${ }^{3,4,14}$ Therefore, self-management interventions were defined as interventions providing information to patients and including minimally two of the following components: 1) stimulation of sign/symptom monitoring, 2) education in problem solving skills (ie, stress/ symptom management), enhancement of 3) medical treatment adherence, 4) physical activity, 5) smoking cessation, or 6) dietary intake. The emphasis for each component had to be on enhancing the patient's active role and responsibility. Therefore, interventions focusing on pulmonary rehabilitation were not considered eligible for this meta-analysis.

Studies were selected by two researchers working independently (NHJ and HW) and included if they 1) met the requirements of the definition of self-management intervention above, 2) had a randomized trial design with concealed allocation to treatment, 3) included patients with an established diagnosis of COPD, 4) compared the self-management intervention to usual care or another self-management intervention, 5) reported data on one or more of the selected outcomes, 6) followed patients for at least 6 months, and 7) were reported in English, Dutch, French, German, Italian, Portuguese, or Spanish.

Methodological quality was assessed by two researchers independently (NHJ and HW) using three relevant criteria from the "Risk of bias" tool from the Cochrane Collaboration: ${ }^{12}$ 1) random concealed allocation to treatment, 2) intentionto-treat analysis, and 3) absence of other major sources of bias (eg, high drop-out rates, risk of contamination). Any discrepancies were solved through consensus with a third researcher (JCAT). Studies that scored a high risk of bias on one or more criteria were defined as "high risk of bias". Those studies were included, but their impact on the results was assessed in a sensitivity analysis. 


\section{Data collection}

The principal investigators of selected studies were invited to participate in this IPD meta-analysis and share their de-identified trial data. Data from each trial were checked on range, extreme values, internal consistency, missing values, and consistency with published reports. Questions that arose during the data checking were discussed and resolved with principal investigators. Details on requested variables, data management, project management, and ethical considerations can be found in the published protocol. ${ }^{13}$

\section{Outcomes}

The main outcomes of this study included: HRQoL at 6 and 12 months (as measured with Chronic Respiratory Questionnaire $^{15}$ or St George Respiratory Questionnaire ${ }^{16}$ ), respiratory-related hospitalization (time-to-first-event, within
6 and 12 months), all-cause hospitalizations (time-to-firstevent, within 6 and 12 months), and mortality (time-to-event, within 6 and 12 months). Additional outcomes analyzed were generic quality of life (QoL), as measured with the Short Form Health Survey, ${ }^{17}$ and total days of respiratory-related and allcause hospital stay since enrollment at 6 and 12 months.

\section{Patient-specific effect modifiers}

Clinically relevant potential effect modifiers (ie, variables, such as sex or age) were prespecified based on the selfmanagement literature and availability of data across trials and presented in Table 1 (along with the baseline data). Based on teleconferences with the principal investigators, we decided to collect data on baseline exacerbation frequency, in addition to the potential effect modifiers prespecified in the protocol. ${ }^{13}$

Table I Baseline characteristics of COPD patients

\begin{tabular}{|c|c|c|c|c|}
\hline Determinant & Categories & Control $(n=I, 492)$ & Intervention $(n=I, 790)$ & Total $(\mathbf{N}=3,282)$ \\
\hline \multirow[t]{2}{*}{ Sex } & Male & $999(67.0)$ & $\mathrm{I}, 15 \mathrm{I}(64.3)$ & $2,150(65.5)$ \\
\hline & Female & $492(33.0)$ & $639(35.7)$ & $\mathrm{I}, 13 \mathrm{I}(34.5)$ \\
\hline \multirow[t]{4}{*}{ Age } & Mean (SD) years & $68.3(9.6)$ & $67.9(9.6)$ & $68.1(9.6)$ \\
\hline & $<65$ years & $487(32.8)$ & $619(34.7)$ & $1,106(33.8)$ \\
\hline & $65-75$ years & $627(42.2)$ & 780 (43.7) & $\mathrm{I}, 407(43.0)$ \\
\hline & $>75$ years & $37 \mid(25.0)$ & $387(21.7)$ & $758(23.2)$ \\
\hline \multirow[t]{3}{*}{ Airflow obstruction } & Mean (SD) $\mathrm{FEV}_{1}$ in \% predicted & $47.3(18.8)$ & $48.0(18.9)$ & $47.7(18.9)$ \\
\hline & $\geq 50 \% \mathrm{FEV}_{1}$ in $\%$ of predicted & $617(42.1)$ & $772(43.5)$ & $1,389(42.9)$ \\
\hline & $<50 \% \mathrm{FEV}_{1}$ in $\%$ of predicted & 847 (57.9) & $\mathrm{I}, 00 \mathrm{I}(56.5)$ & $\mathrm{I}, 848(57.1)$ \\
\hline \multirow[t]{2}{*}{ Dyspnea $^{a}$} & Low level of breathlessness & I5I (37.4) & $275(50.2)$ & $426(44.7)$ \\
\hline & High level of breathlessness & $253(62.6)$ & $273(49.8)$ & $526(55.3)$ \\
\hline \multirow[t]{3}{*}{ Comorbidity index } & No comorbid conditions & $366(32.4)$ & $428(32.9)$ & $794(32.7)$ \\
\hline & Comorbid conditions in one cluster & $291(25.8)$ & $326(25.0)$ & $617(25.4)$ \\
\hline & Comorbid conditions in $\geq 2$ clusters & $47 \mid(4 I .8)$ & $548(42.1)$ & $1,019(41.9)$ \\
\hline \multirow[t]{2}{*}{ Depression $^{c}$} & No/mild depression & $4 \mid 4(70.9)$ & $546(74.0)$ & $960(72.6)$ \\
\hline & Moderate/severe depression & $170(29.1)$ & $192(26.0)$ & $362(27.4)$ \\
\hline \multirow[t]{3}{*}{ Level of education } & Primary education or below & $313(39.6)$ & $391(38.3)$ & $704(38.9)$ \\
\hline & Secondary education & $35 \mathrm{I}(44.4)$ & $456(44.7)$ & $807(44.6)$ \\
\hline & Higher education & $127(16.1)$ & $173(17.0)$ & $300(16.6)$ \\
\hline \multirow[t]{3}{*}{ Self-efficacy ${ }^{d}$} & Low self-efficacy & $185(31.7)$ & $290(33.9)$ & $475(33.0)$ \\
\hline & Moderate self-efficacy & $216(37.0)$ & $268(31.3)$ & $484(33.6)$ \\
\hline & High self-efficacy & $183(31.3)$ & $297(34.7)$ & $480(33.4)$ \\
\hline \multirow[t]{2}{*}{ Living status } & Living with others & $548(71.2)$ & $572(68.1)$ & $1,120(69.6)$ \\
\hline & Living alone & $222(28.8)$ & $268(31.9)$ & $490(30.4)$ \\
\hline \multirow[t]{4}{*}{ Body mass index } & Mean (SD) & $27.2(6.5)$ & $27.1(6.2)$ & $27.1(6.3)$ \\
\hline & $<25$ & $539(40.1)$ & $64 I(39.4)$ & $1,180(39.7)$ \\
\hline & $25-29.99$ & $429(31.9)$ & $568(34.9)$ & $997(33.6)$ \\
\hline & $\geq 30$ & $376(28.0)$ & $418(25.7)$ & $794(26.7)$ \\
\hline \multirow[t]{2}{*}{ Smoking status } & Current nonsmoker & I,036 (7I.8) & I,225 (7I.I) & $2,26 \mid(71.4)$ \\
\hline & Current smoker & $407(28.2)$ & $499(28.9)$ & $906(28.6)$ \\
\hline \multirow[t]{3}{*}{ Exacerbation frequency } & 0 exacerbations & $188(3 \mid .1)$ & $194(30.8)$ & $382(31.0)$ \\
\hline & I exacerbation & $134(22.2)$ & $122(19.4)$ & $256(20.7)$ \\
\hline & $\geq 2$ exacerbations & $282(46.7)$ & $3 \mid 4(49.8)$ & $596(48.3)$ \\
\hline
\end{tabular}

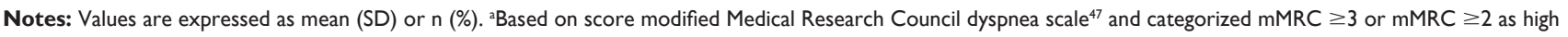
level of breathlessness. 'Based on clusters Cumulative Illness Rating Scale. ${ }^{48}$ Based on validated cutoff scores of instrument used in each specific study. ${ }^{\mathrm{d}} \mathrm{Categories} \mathrm{based} \mathrm{on}$ tertile scores computed within each specific study.

Abbreviations: $\mathrm{FEV}_{1}$, forced expiratory volume in I second; SD, standard deviation. 


\section{Statistical analysis}

The principal investigators of the individual trials were involved in the process of designing a detailed plan for statistical analysis and agreed upon this prior to data analysis. Missing values were imputed within studies only using multiple imputation by chained equations (overall $2.7 \%$ missing data, except $33.7 \%$ for HRQoL follow-up data). ${ }^{18}$ For each study, 25 multiple imputed datasets were created and used for the primary analyses. Within these analyses, a one-stage approach was used, that is, simultaneously analyzing all observations while accounting for clustering of observations within studies. ${ }^{19}$ Results of imputed datasets were pooled using Rubin's rules. ${ }^{20}$

All analyses were carried out according to the intentionto-treat principle. For time-to-event endpoints, effects of self-management were quantified by estimating hazard ratios using Cox proportional hazard models, including a frailty term to account for clustering within studies. The continuous outcomes (HRQoL and generic $\mathrm{oL}$ ) were quantified by standardized mean differences (SMD) between intervention arms and analyzed using linear mixed effects models. Using the SMD, results are converted to a uniform scale representing the intervention effect relative to the observed variability in one study before pooling the results of different studies. Binary outcome data (mortality, respiratory-related, and all-cause hospitalization) were analyzed with log-binomial mixed effects models, which estimated risk ratios (RRs) or odds ratios (ORs) in case of nonconvergence of a model, respectively. To correctly model the presence of overdispersion in the count data of total days of hospital stay, negative binomial mixed effects models were used to estimate relative length of stay. All mixed effects models included a random intercept and a random slope for the treatment effect to take clustering at study level into account.

To assess whether the effect of self-management was modified by patient characteristics, the aforementioned models were extended with interaction terms for the patient characteristics included in Table 1. The independent variables in the models were random intercept, random slope, allocation to self-management, patient characteristic, and interaction term (treatment allocation*patient characteristic). This was performed for each patient characteristic separately. All effect modifiers with $P<0.10$ for the interaction (likelihood ratio test) in the univariable analysis were included in a multivariable model to estimate the effect of self-management within subgroups independent of other potential effect modifiers. Effect modification was considered significant if the interaction term showed $P<0.05$ in the final model.
As a sensitivity analysis, we investigated the potential of retrieval bias (ie, bias due to selective inclusion of studies in the IPD meta-analysis) by pooling the published main effects of studies for which IPD were unavailable with the main effects of included studies in a random effects meta-analysis. To assess the impact of studies of lower methodological quality on the main effects, an additional sensitivity analysis was performed, including only studies with a low risk of bias. Three additional sensitivity analyses were performed to assess the robustness of findings from the subgroup analyses: 1) a complete case analysis was carried out to assess the effect of imputing data, and analyses were repeated by 2) excluding older studies (recruitment before 2001) and 3) excluding the largest trial. ${ }^{6}$ All analyses were performed in $\mathrm{R}$ for Windows version 3.1.1 (R Development Core Team. Released 2013. Vienna, Austria: R Foundation for Statistical Computing).

\section{Results}

Twenty-one studies met the inclusion criteria. Seven studies were not included in this IPD meta-analysis. ${ }^{9,21-26} \mathrm{We}$ could not contact the investigators of three studies, ${ }^{21,23,24}$ for two studies, the investigators could not obtain approval from their local Institutional Review Board; ${ }^{9,22}$ the data from one study were no longer available, ${ }^{25}$ and investigators of one study could not participate due to time constraints. ${ }^{26}$ The investigators of the other 14 studies participated in this IPD meta-analysis, resulting in the inclusion of data on 3,282 patients.

Patient characteristics are presented in Table 1. Not all studies measured the same baseline characteristics; only sex, age, and forced expiratory volume in 1 second in $\%$ of predicted were assessed in all studies. The majority of included patients were male $(65.5 \%)$. Patients had a mean age of 68.1 years $( \pm 9.6)$ and a mean forced expiratory volume in 1 second in $\%$ of predicted of $47.7 \%( \pm 18.9 \%)$. The majority of patients had high breathlessness scores (55.3\%). Apart from dyspnea classification, all baseline variables were well balanced between control and intervention groups.

Table 2 presents the characteristics of included studies. ${ }^{5-8,27-36}$ Seven studies recruited participants in a clinic or hospital setting,,$^{5-7,27,29,30,32}$ five studies in general practice,,$^{8,28,31,34,36}$ and two in both settings. ${ }^{33,35}$ The sample size of studies ranged from $53^{30}$ to 743 patients. ${ }^{6}$ Self-management interventions varied across studies: a majority included an action plan and consisted of individual sessions with a nurse, and some involved group contacts. Duration of interventions ranged from 1 day $^{31}$ to 24 months. ${ }^{8}$ 


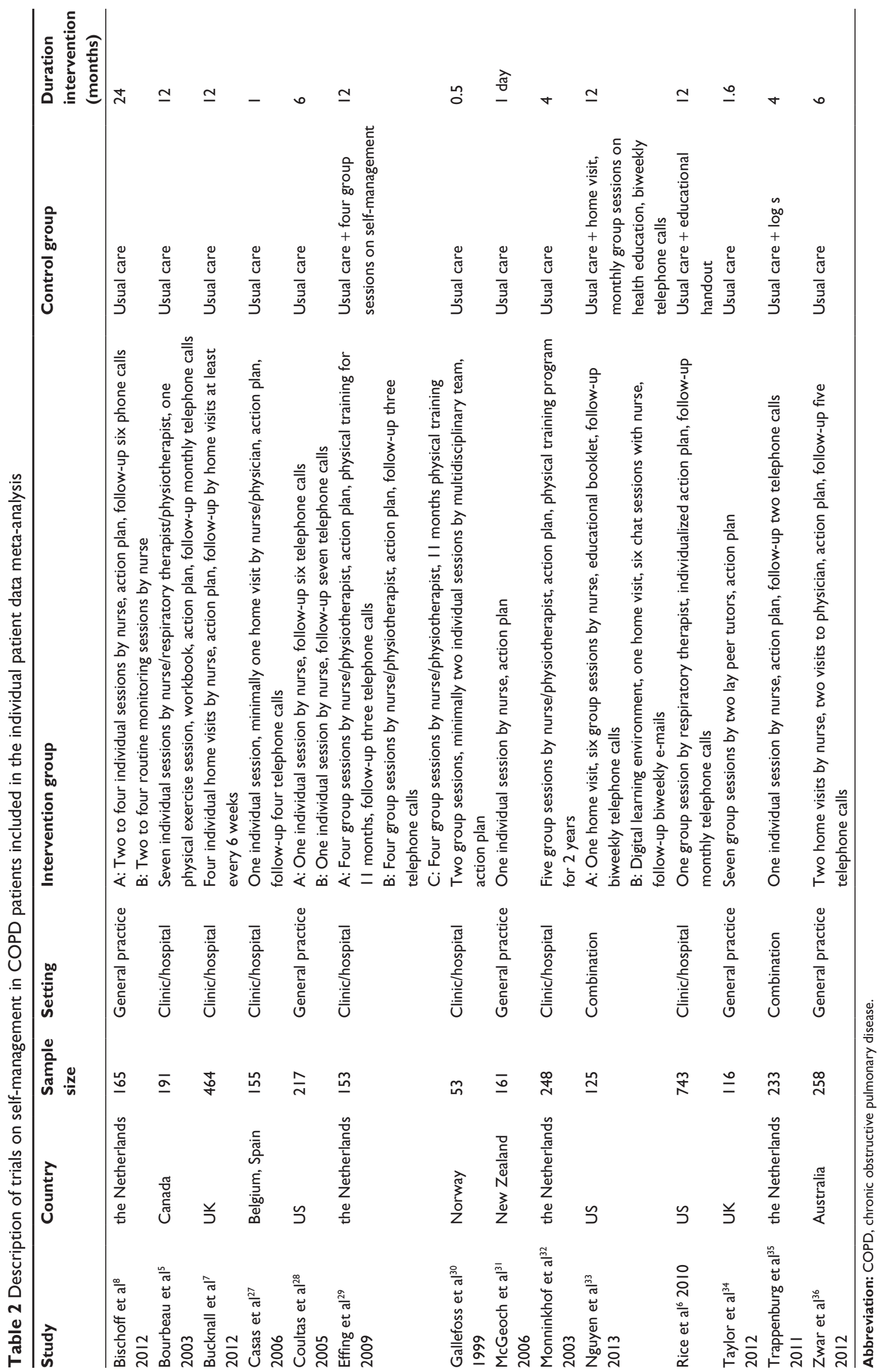




\section{Main effects of self-management interventions}

Self-management interventions improved HRQoL at 12 months (SMD 0.08, 95\% confidence interval [CI] $0.00-0.16$ ), but not at 6 months (SMD $0.05,95 \%$ CI -0.05 to 0.15 ) (Table 3). The interventions improved time to first respiratory-related hospitalization (hazard ratio $0.79,95 \% \mathrm{CI}$ 0.66-0.94). Although there was no clear effect on respiratoryrelated hospitalization within 6 months, there was a significant risk reduction at 12 months (RR $0.77,95 \%$ CI $0.64-0.93$ ). Self-management interventions improved the time to first allcause hospitalization (hazard ratio $0.80,95 \%$ CI $0.69-0.90$ ) and risk of hospitalization within 6 months (RR $0.81,95 \%$ CI 0.67-0.97) and 12 months (RR 0.84, 95\% CI 0.73-0.96). There was no effect of self-management on mortality. Figure 1 shows the effects across studies for HRQoL, respiratoryrelated and all-cause hospitalization, and mortality. Sensitivity analyses of including the published effects of studies for which no IPD were available resulted in similar effects of the self-management interventions (Supplementary material). No effects were observed on the additional outcomes of generic QoL or total days in hospital (Supplementary material).

\section{Effects in subgroups of patients}

The final models in the prespecified subgroup analysis revealed no consistent effect modification by any patient characteristic across all relevant outcomes (Table 4), but the effect on specific outcomes differed according to some of the patient characteristics we studied. A positive effect of self-management interventions was observed in males (OR $0.61,95 \%$ CI $0.41-0.90$ ) compared to females on the outcome respiratory-related hospitalization within 6 months (OR 1.24, 95\% CI 0.76-2.02; interaction $P=0.006$ ). Patients with severe airflow limitation showed a reduced risk on allcause hospitalization within 6 months when allocated to the intervention (RR $0.71,95 \%$ CI 0.58-0.88), while there was no treatment effect in patients with $\geq 50 \%$ forced expiratory volume in 1 second in \% of predicted (RR 1.02, 95\% CI 0.78-1.34; interaction $P=0.016$ ). Obese patients showed the most protective effects of self-management interventions on respiratory-related hospitalization within 6 months (OR $0.44,95 \%$ CI $0.27-0.72$; interaction $P=0.038$ ) and mortality within 6 months (OR $0.35,95 \%$ CI $0.11-1.10$; interaction $P=0.026$ ). Finally, patients with baseline moderate self-efficacy scores showed the largest reduction in risk on respiratory-related hospitalization within 12 months (OR 0.39, 95\% CI 0.21-0.75) compared to patients with low (OR $0.85,95 \%$ CI $0.46-1.59)$ or high levels of selfefficacy (OR 0.89, 95\% CI 0.47-1.71; interaction $P=0.036$ ). Additional analyses for generic QoL and total days in hospital did not reveal different insights (Supplementary material). Subgroup analysis according to exacerbation frequency was impossible due to too diverse data collection at baseline and comparison of subgroups in individual trials did not reveal consistent effects across studies (Supplementary material). Sensitivity analyses to assess the robustness of the subgroup effects yielded similar findings to the primary analysis.

\section{Discussion}

This IPD meta-analysis of 14 randomized trials showed that self-management interventions exerted positive effects in COPD patients on respiratory-related and all-cause

Table 3 Effects of self-management interventions in patients with COPD

\begin{tabular}{|c|c|c|c|c|c|}
\hline Outcome & $\mathbf{N}$ studies & n patients & Effect measure & Treatment effect & $95 \% \mathrm{Cl}$ \\
\hline \multicolumn{6}{|c|}{ Health-related quality of life } \\
\hline At 6 months & 9 & $\mathrm{I}, 876$ & SMD & 0.05 & $(-0.05-0.15)$ \\
\hline At 12 months & 10 & 2,663 & SMD & 0.08 & $(0.00-0.16)$ \\
\hline \multicolumn{6}{|c|}{ Respiratory-related hospitalization } \\
\hline Time-to-first-event & 6 & $\mathrm{I}, 872$ & $H R$ & 0.79 & $(0.66-0.94)$ \\
\hline Within 6 months & 8 & 2,347 & $\mathrm{RR}$ & 0.87 & $(0.69-1.09)$ \\
\hline Within 12 months & 9 & 2,426 & RR & 0.77 & $(0.64-0.93)$ \\
\hline \multicolumn{6}{|l|}{ All-cause hospitalization } \\
\hline Time-to-first-event & 4 & 1,559 & $\mathrm{HR}$ & 0.80 & $(0.69-0.93)$ \\
\hline Within 6 months & 6 & 2,034 & $\mathrm{RR}$ & 0.81 & $(0.67-0.97)$ \\
\hline Within 12 months & 5 & 1,817 & RR & 0.84 & $(0.73-0.96)$ \\
\hline \multicolumn{6}{|l|}{ Mortality } \\
\hline Time-to-event & 7 & 2,120 & $\mathrm{HR}$ & 1.02 & $(0.76-1.37)$ \\
\hline Within 6 months & 9 & 2,490 & $\mathrm{RR}$ & 1.06 & $(0.62-1.82)$ \\
\hline Within 12 months & 7 & 2,182 & RR & 1.04 & $(0.64-1.69)$ \\
\hline
\end{tabular}

Abbreviations: $\mathrm{Cl}$, confidence interval; $\mathrm{HR}$, hazard ratio; RR, risk ratio; SMD, standardized mean difference. 


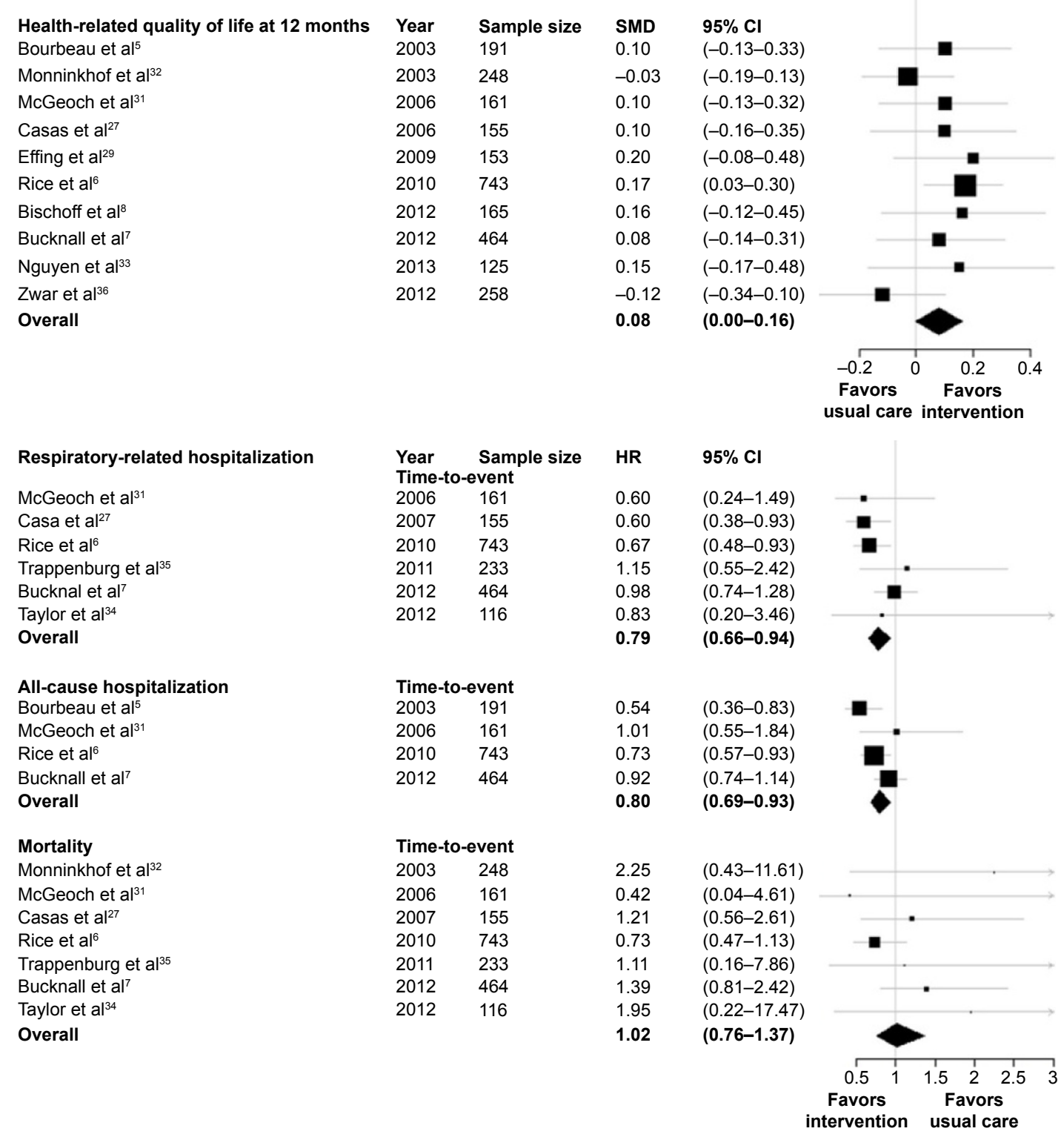

Figure I Forest plot of effects of self-management interventions on health-related quality of life, respiratory-related and all-cause hospitalization, and mortality in patients with COPD.

Abbreviations: $\mathrm{Cl}$, confidence interval; $\mathrm{HR}$, hazard ratio, $\mathrm{SMD}$, standardized mean difference.

hospitalization. Self-management interventions also resulted in small improvements on HRQoL at 12 months, but had no effect on HRQoL at 6 months or on mortality. One novel aspect from this study was the prespecified subgroup analyses, which did not show a consistent pattern across health outcomes of subgroups of patients benefiting most from the self-management interventions.

The main effects reported by the present study are in line with a recent Cochrane review on self-management trials in COPD patients. ${ }^{4}$ Like the present study, the authors did not find an effect of self-management on mortality. However, the follow-up period of 12 months may have been too short to elicit an effect on this outcome. Although the Cochrane review applied a wider definition of "self-management interventions" and could include all eligible trials ( $\mathrm{N}=23$ vs $\mathrm{N}=14$ in this IPD meta-analysis, respectively), we were able to include more recently conducted studies $(\mathrm{N}=6)$ of which some have cast doubts on the usefulness of self-management in COPD patients. ${ }^{7,8}$ By including data from these recent studies as well as performing a sensitivity analysis, including the published results of the prematurely terminated trial, ${ }^{9}$ the present study provides more extensive evidence that self-management interventions elicit positive effects in COPD patients and can be considered safe. However, the 
Table 4 Effects of self-management interventions in subgroups of patients with COPD

\begin{tabular}{|c|c|c|c|c|c|c|c|c|c|}
\hline \multirow[t]{2}{*}{ Outcome } & \multirow{2}{*}{$\begin{array}{l}\text { n } \\
\text { patients }\end{array}$} & \multirow[t]{2}{*}{ Subgroup } & \multirow{2}{*}{$\begin{array}{l}\text { Effect } \\
\text { measure }\end{array}$} & \multicolumn{3}{|c|}{ Univariable analysis } & \multicolumn{3}{|c|}{ Multivariable analysis } \\
\hline & & & & $\begin{array}{l}\text { Treatment } \\
\text { effect }\end{array}$ & $95 \% \mathrm{Cl}$ & $\begin{array}{l}P \text {-value for } \\
\text { interaction }\end{array}$ & $\begin{array}{l}\text { Treatment } \\
\text { effect }\end{array}$ & $95 \% \mathrm{Cl}$ & $\begin{array}{l}P \text {-value for } \\
\text { interaction }\end{array}$ \\
\hline \multicolumn{10}{|c|}{ Health-related quality of life } \\
\hline At 6 months & & \multicolumn{8}{|l|}{ No subgroup effects } \\
\hline At 12 months & & \multicolumn{8}{|l|}{ No subgroup effects } \\
\hline \multicolumn{10}{|c|}{ Respiratory-related hospitalization } \\
\hline \multirow{7}{*}{ Time-to-first-event } & $\mathrm{I}, 2 \mathrm{|} 4$ & Males & $\mathrm{HR}$ & 0.68 & $(0.54-0.84)$ & 0.022 & 0.78 & $(0.55-1.09)$ & 0.130 \\
\hline & 497 & Females & & 1.05 & $(0.78-1.42)$ & & 1.07 & $(0.78-1.48)$ & \\
\hline & 549 & No comorbidities & $\mathrm{HR}$ & 0.93 & $(0.72-1.20)$ & 0.073 & 0.78 & $(0.55-1.09)$ & 0.206 \\
\hline & 380 & Comorbidities & & 0.52 & $(0.33-0.80)$ & & 0.48 & $(0.31-0.76)$ & \\
\hline & & in one cluster & & & & & & & \\
\hline & 710 & Comorbidities & & 0.72 & $(0.52-1.00)$ & & 0.70 & $(0.50-0.97)$ & \\
\hline & & in $>$ I cluster & & & & & & & \\
\hline \multirow[t]{5}{*}{6 months } & $\mathrm{I}, 544$ & Males & OR & 0.67 & $(0.50-0.88)$ & 0.005 & 0.61 & $(0.4 I-0.90)$ & 0.006 \\
\hline & 803 & Females & & 1.36 & $(0.91-2.04)$ & & 1.24 & $(0.76-2.02)$ & \\
\hline & 835 & BMI $<25$ & OR & 0.77 & $(0.54-1.11)$ & 0.043 & 0.61 & $(0.4 I-0.90)$ & 0.038 \\
\hline & 636 & BMI 25-29.99 & & 1.23 & $(0.78-1.93)$ & & 1.01 & $(0.63-1.61)$ & \\
\hline & 618 & $\mathrm{BMI} \geq 30$ & & 0.55 & $(0.34-0.88)$ & & 0.44 & $(0.27-0.72)$ & \\
\hline \multirow[t]{4}{*}{12 months } & 281 & Low self-efficacy & OR & 0.85 & $(0.46-1.59)$ & 0.036 & a & & \\
\hline & 277 & Moderate & & 0.39 & $(0.21-0.75)$ & & & & \\
\hline & & self-efficacy & & & & & & & \\
\hline & 250 & High self-efficacy & & 0.89 & $(0.47-I .7 I)$ & & & & \\
\hline \multicolumn{10}{|c|}{ All-cause hospitalization } \\
\hline \multirow{4}{*}{ Time-to-first-event } & 539 & No comorbidities & $H R$ & 0.88 & $(0.7 I-1.09)$ & 0.056 & a & & \\
\hline & 384 & $\begin{array}{l}\text { Comorbidities in } \\
\text { one cluster }\end{array}$ & & 0.57 & $(0.4 I-0.78)$ & & & & \\
\hline & 636 & Comorbidities & & 0.89 & $(0.68-1.15)$ & & & & \\
\hline & & in $>$ I cluster & & & & & & & \\
\hline \multirow[t]{4}{*}{6 months } & 698 & $\geq 50 \% \mathrm{FEV}_{1}$ & $\mathrm{RR}$ & 1.02 & $(0.78-1.34)$ & 0.016 & a & & \\
\hline & & $\%$ predicted & & & & & & & \\
\hline & 1,336 & $<50 \% \mathrm{FEV}_{1}$ & & 0.71 & $(0.58-0.88)$ & & & & \\
\hline & & $\%$ predicted & & & & & & & \\
\hline 12 months & 614 & $\geq 50 \% \mathrm{FEV}_{1}$ & OR & 1.03 & $(0.73-1.46)$ & 0.032 & 1.30 & $(0.54-3.12)$ & 0.232 \\
\hline & & $\%$ predicted & & & & & & & \\
\hline & 1,203 & $<50 \% \mathrm{FEV}_{1}$ & & 0.63 & $(0.49-0.81)$ & & 0.82 & $(0.40-1.68)$ & \\
\hline & & $\%$ predicted & & & & & & & \\
\hline & 222 & Low self-efficacy & OR & 0.93 & $(0.47-1.86)$ & 0.047 & 1.30 & $(0.54-3.12)$ & 0.062 \\
\hline & 239 & Moderate & & 0.35 & $(0.18-0.71)$ & & 0.52 & $(0.21-1.29)$ & \\
\hline & & self-efficacy & & & & & & & \\
\hline & 195 & High self-efficacy & & 0.92 & $(0.46-1.86)$ & & 1.31 & $(0.54-3.20)$ & \\
\hline Mortality & & & & & & & & & \\
\hline Time-to-event & 549 & No comorbidities & $\mathrm{HR}$ & 1.56 & $(0.90-2.72)$ & 0.075 & b & & \\
\hline & 380 & Comorbidities & & 0.66 & $(0.29-1.49)$ & & & & \\
\hline & & in one cluster & & & & & & & \\
\hline & 710 & Comorbidities & & 0.77 & $(0.46-\mid .31)$ & & & & \\
\hline & & in $>$ I cluster & & & & & & & \\
\hline & 140 & Less & $\mathrm{HR}$ & 5.33 & $(0.69-40.91)$ & 0.091 & b & & \\
\hline & & breathlessness & & & & & & & \\
\hline & 248 & More & & 0.83 & $(0.22-3.09)$ & & & & \\
\hline & & breathlessness & & & & & & & \\
\hline 6 months & $\mathrm{I}, 707$ & Males & OR & 0.79 & $(0.42-1.49)$ & 0.071 & 1.40 & $(0.64-3.05)$ & 0.123 \\
\hline & 783 & Females & & 1.83 & $(0.82-4.11)$ & & 2.89 & (1.19-7.02) & \\
\hline & 976 & $\mathrm{BMI}<25$ & OR & 1.83 & $(0.94-3.55)$ & 0.016 & 1.40 & $(0.64-3.05)$ & 0.026 \\
\hline & 817 & BMI 25-29.99 & & 0.70 & $(0.33-I .5 I)$ & & 0.54 & $(0.23-1.26)$ & \\
\hline & 697 & $\mathrm{BMI} \geq 30$ & & 0.46 & $(0.15-1.35)$ & & 0.35 & $(0.1 I-I .10)$ & \\
\hline 12 months & I,557 & Males & $\mathrm{RR}$ & 0.85 & $(0.5 \mathrm{I}-\mathrm{I} .42)$ & 0.092 & 1.18 & $(0.62-2.26)$ & 0.445 \\
\hline & 625 & Females & & 1.59 & $(0.77-3.28)$ & & 1.57 & $(0.80-3.08)$ & \\
\hline
\end{tabular}


Table 4 (Continued)

\begin{tabular}{|c|c|c|c|c|c|c|c|c|c|}
\hline \multirow[t]{2}{*}{ Outcome } & \multirow{2}{*}{$\begin{array}{l}\text { n } \\
\text { patients }\end{array}$} & \multirow[t]{2}{*}{ Subgroup } & \multirow{2}{*}{$\begin{array}{l}\text { Effect } \\
\text { measure }\end{array}$} & \multicolumn{3}{|c|}{ Univariable analysis } & \multicolumn{3}{|c|}{ Multivariable analysis } \\
\hline & & & & $\begin{array}{l}\text { Treatment } \\
\text { effect }\end{array}$ & $95 \% \mathrm{Cl}$ & $\begin{array}{l}P \text {-value for } \\
\text { interaction }\end{array}$ & $\begin{array}{l}\text { Treatment } \\
\text { effect }\end{array}$ & $95 \% \mathrm{Cl}$ & $\begin{array}{l}P \text {-value for } \\
\text { interaction }\end{array}$ \\
\hline & 627 & No Comorbidities & $\mathrm{RR}$ & 1.37 & $(0.80-2.35)$ & 0.074 & 1.18 & $(0.62-2.26)$ & 0.213 \\
\hline & 485 & Comorbidities & & 0.64 & $(0.31-1.30)$ & & 0.60 & $(0.30-1.25)$ & \\
\hline & & in I cluster & & & & & & & \\
\hline & 669 & Comorbidities & & 0.71 & $(0.43-1.16)$ & & 0.70 & $(0.43-1.15)$ & \\
\hline & & in $>$ I cluster & & & & & & & \\
\hline
\end{tabular}

Notes: Results of the subgroup analyses are only presented if a potential effect modifier showed an effect with $P<0.10$ in the univariable analysis. ${ }^{a}$ Multivariable analysis was only performed if $\geq 2$ potential effect modifiers in the univariable analysis to adjust for other potential effect modifiers. ${ }^{b}$ Multivariable analysis not executed as this would result in $\mathrm{N}=$ I study.

Abbreviations: $\mathrm{BMI}$, body mass index; $\mathrm{Cl}$, confidence interval; COPD, chronic obstructive pulmonary disease; $\mathrm{FEV}_{\mathrm{l}}$, forced expiratory volume in I second; HR, hazard ratio; $O R$, odds ratio; $R R$, risk ratio.

positive effects observed for HRQoL at 12 months should be considered modest improvements, and no effects were observed at 6 months. It also remains questionable whether the statistical difference that we observed is a clinically important difference for COPD patients. Furthermore, it remains questionable whether our findings also apply to COPD patients recently discharged from hospital. A recently published systematic review on self-management interventions in this group of patients found that positive effects were limited to HRQoL, ${ }^{37}$ but the authors applied rather wide inclusion criteria for the interventions, resulting in the inclusion of many interventions with only a limited self-management component compared to the present study.

The novel aspect of the present study compared to the previously conducted systematic reviews was the prespecified subgroup analysis. This subgroup analysis revealed larger effects of self-management interventions in males, patients with more severe airflow limitation, patients with moderate levels of self-efficacy, and obese patients, but only on some outcomes. To date, differential effects of selfmanagement interventions in subgroups of COPD patients have scarcely been examined. One study included in this IPD meta-analysis analyzed response of subgroups of COPD patients to the self-management intervention on hospitalization or death. ${ }^{7}$ The preplanned subgroup analyses did not show any evidence of differential effects, but the authors found that only $42 \%$ of intervention group subjects learnt to self-manage successfully. The successful self-managing patients had significantly reduced hospitalization rate. ${ }^{7}$ The present IPD meta-analysis, with more power to perform subgroup analyses, suggested larger effects of self-management interventions on respiratory-related hospitalization as well as mortality at 6 months in obese patients. Although effect modification by body mass index has not yet been analyzed in COPD patients in the context of self-management interventions, evidence is starting to emerge that overweight or obese patients encompass a specific phenotype of COPD patients. ${ }^{38}$ It is possible that this particular phenotype of COPD patients responds differently to self-management interventions. Our analyses only revealed differential effects of obesity on the outcomes respiratory-related hospitalization at 6 months and mortality at 6 months. Effects at 12 months were in a similar direction, but these were not statistically significant. Previous efforts to assess the influence of body mass index on effectiveness of pulmonary rehabilitation have also yielded inconsistent results. ${ }^{39,40}$ Although our subgroup analysis was prespecified ${ }^{13}$ and yielded several statistically significant findings, the high number of analyses increases the chance of false-positive findings. With no consistent pattern across multiple health outcomes, the subgroup results should be interpreted with caution. ${ }^{41}$ Limiting self-management support to specific patient subgroups cannot be recommended at this stage and further research is therefore needed to confirm the observed subgroup effects for other health outcomes. Reassuringly, there were no indications in our analyses that certain subgroups of patients responded in a negative way to the self-management interventions.

To our knowledge, the present study is the first to pool and reanalyze the original data of a large number of randomized trials on self-management interventions in patients with COPD and transcends the previously conducted systematic reviews. ${ }^{4,37}$ An IPD meta-analysis is a resource intensive approach, given the time and efforts needed for collecting and merging the raw patient data. ${ }^{42}$ As a result, no articles published after June 2013 were included for analysis. The high response rate of principal investigators $(66.7 \%)$, large number of patients included $(n=3,282)$, prespecified statistical plan, and close collaboration with the principal investigators through regular teleconferences contribute to the robustness of our findings. There are several methodological limitations worth considering. First, in spite of numerous efforts to contact and convince the principal investigators of all eligible 
studies, we could not obtain the data of seven randomized trials, including the prematurely aborted trial. ${ }^{9}$ However, the sensitivity analysis of pooling the published results of those trials with the main effects of included studies showed that this did not alter our findings (Supplementary material).

Second, we assumed all interventions to be homogeneous self-management interventions in our analyses, but the included self-management intervention designs differed from each other in terms of dose, mode, and content. Without consistent evidence for subgroups of patients benefiting across various health outcomes, we could hypothesize that specific subgroups of patients only respond better to particular components of interventions (ie, action plans in self-management interventions). Future research addressing various interventions is needed to identify what type of intervention works for whom. Nevertheless, the reported main effects on HRQoL at 12 months, and respiratory-related and all-cause hospitalization were consistent across cultures and health care settings. This indicates that, despite their diversity, self-management interventions exert positive effects, even in different formats and differing patient populations.

Third, this IPD meta-analysis was highly dependent on data that were previously collected. This seriously limited our choice of potential effect modifiers. Exacerbation frequency has attracted considerable attention in recent years, ${ }^{43}$ but due to the diverse data collection across studies, the quality of available data on baseline exacerbation rate was too low to enable a pooled analysis of this patient characteristic. This emphasizes the urgent need for a uniform operational definition of exacerbations within the field of COPD research. ${ }^{44}$ For similar reasons, we could not study other potentially relevant variables, such as Global initiative for chronic Obstructive Lung Disease stage, coping style, disease perception, and adherence. Previous studies have shown that even though adherence to self-management treatment is a challenge for a majority of patients enrolled in randomized trials, the patients who actually applied those new self-management skills showed better outcomes. 7,45 This suggests that emphasis should be placed on patients' ability to apply self-management guidelines and subsequently change their behavior as this is a prerequisite for better outcomes. Collection of data on intervention delivery, treatment adherence, and behavior change in randomized trials, particularly on complex interventions, such as selfmanagement, is indispensable to identify patients most likely to adhere to the self-management interventions and in whom these interventions may improve prognosis. ${ }^{46}$

\section{Conclusion}

Self-management interventions exert positive effects in patients with COPD on respiratory-related and all-cause hospitalization and modest improvement of HRQoL at 12 months, but do not show an effect on mortality. These benefits seem similar across the subgroups of patients studied as subgroup analysis did not reveal a consistent pattern across different health outcomes. Our findings support implementation of self-management strategies in practice, but targeting self-management interventions at specific subgroups of patients cannot be recommended based on the current evidence.

\section{Author contributions}

NHJ, HW, JCAT, RHHG, JB, TWE, JvdP, TT, AWH, and MJS participated in the design of the study. JCAT, EWMAB, JB, CEB, DC, TWE, ME, FG, JG-A, EMM, HQN, KLR, MS, SJCT, and NAZ contributed data to this study. NHJ and HW collected and merged the data. NHJ, HW, JCAT, RHHG, AWH, and MJS wrote the statistical analysis plan. NHJ and RHHG carried out the statistical analysis. All authors reviewed and approved the statistical plan and contributed to the interpretation of the statistical analysis. NHJ wrote the draft of the manuscript. All authors contributed to the critical revision of the manuscript. All authors contributed toward data analysis, drafting and revising the paper and agree to be accountable for all aspects of the work.

\section{Disclosure}

The authors declare the following interests: this work was supported by a grant from the Netherlands Organisation for Health Research and Development, ZonMw (grant number 520001002). The funding source had no involvement in the design of this study, in the writing of the report, and in the decision to submit the paper for publication. JB reports grants from AstraZeneca, Boehringer Ingelheim, Canadian Respiratory Research Network, Novartis, GlaxoSmithKline, Takeda, Pfizer, and Grifols, all outside the submitted work. DC received grant funding from the National Institutes of Health, outside the submitted work. KLR reports grants from Veterans Affairs VISN 23, nonfinancial support from Research Service Minneapolis Veterans Affairs Health Care Service, and grants from GlaxoSmithKline, all outside the submitted work. NAZ reports grants from Australian National Health and Medical Research Council, outside the submitted work. The authors report no further conflicts of interest in this work. 


\section{References}

1. Mathers C, Boerma T, Ma Fat D. The Global Burden of Disease: 2004 Update. Geneva, Switzerland: World Health Organization; 2008.

2. Global Initiative for Chronic Obstructive Lung Disease (GOLD). Global strategy for the diagnosis, management and prevention of COPD. GOLD, 2015. Available from: www.goldcopd.org. Accessed October 26, 2015.

3. Bourbeau J, van der Palen J. Promoting effective self-management programmes to improve COPD. Eur Respir J. 2009;33:461-463.

4. Zwerink M, Brusse-Keizer M, van der Valk PD, et al. Self management for patients with chronic obstructive pulmonary disease. Cochrane Database Syst Rev. 2014;3:CD002990.

5. Bourbeau J, Julien M, Maltais F, et al. Reduction of hospital utilization in patients with chronic obstructive pulmonary disease: a disease-specific self-management intervention. Arch Intern Med. 2003;163:585-591.

6. Rice KL, Dewan N, Bloomfield HE, et al. Disease management program for chronic obstructive pulmonary disease: a randomized controlled trial. Am J Respir Crit Care Med. 2010;182:890-896.

7. Bucknall CE, Miller G, Lloyd SM, et al. Glasgow supported selfmanagement trial (GSuST) for patients with moderate to severe COPD randomised controlled trial. BMJ. 2012;344:e1060.

8. Bischoff EW, Akkermans R, Bourbeau J, et al. Comprehensive self management and routine monitoring in chronic obstructive pulmonary disease patients in general practice: randomised controlled trial. $B M J$. 2012;345:e7642.

9. Fan VS, Gaziano JM, Lew R, et al. A comprehensive care management program to prevent chronic obstructive pulmonary disease hospitalizations a randomized, controlled trial. Ann Intern Med. 2012;156:673-683

10. Nici L, Bontly TD, Zuwallack R, Gross N. Self-management in chronic obstructive pulmonary disease. Time for a paradigm shift? Ann Am Thorac Soc. 2014;11:101-107.

11. Riley RD, Lambert PC, Abo-Zaid G. Meta-analysis of individual participant data: rationale, conduct, and reporting. BMJ. 2010;340:c221.

12. Higgins JPT, Green S. Cochrane Handbook for Systematic Reviews of Interventions, version 5.1.0. The Cochrane Collaboration; 2011.

13. Jonkman NH, Westland H, Trappenburg JC, et al. Towards tailoring of self-management for patients with chronic heart failure or chronic obstructive pulmonary disease: a protocol for an individual patient data meta-analysis. BMJ Open. 2014;4:e005220.

14. McGowan PT. Self-management education and support in chronic disease management. Prim Care. 2012;39:307-325.

15. Guyatt GH, Berman LB, Townsend M, Pugsley SO, Chambers LW. A measure of quality of life for clinical trials in chronic lung disease. Thorax. 1987;42:773-778.

16. Jones PW, Quirk FH, Baveystock CM, Littlejohns P. A self-complete measure of health status for chronic airflow limitation. The St. George's Respiratory Questionnaire. Am Rev Respir Dis. 1992;145:1321-1327.

17. Ware JE, Sherbourne CD. The MOS 36-item short-form health survey (SF-36). I. Conceptual framework and item selection. Med Care. 1992; 30:473-483.

18. Groothuis-Oudshoorn K, van Buuren S. MICE: Multivariate imputation by chained equations in R. J Stat Softw. 2011;45(3):1-67.

19. Simmonds MC, Higgins JP, Stewart LA, Tierney JF, Clarke MJ, Thompson SG. Meta-analysis of individual patient data from randomized trials: a review of methods used in practice. Clin Trials. 2005;2 209-217.

20. Rubin DB. Multiple Imputation for Non-response in Surveys. New York: John Wiley \& Sons; 1987.

21. Khdour MR, Kidney JC, Smyth BM, McElnay JC. Clinical pharmacy-led disease and medicine management programme for patients with COPD. Br J Clin Pharmacol. 2009;68:588-598.

22. Ninot G, Moullec G, Picot MC, et al. Cost-saving effect of supervised exercise associated to COPD self-management education program. Respir Med. 2011;105:377-385.

23. Rea H, McAuley S, Stewart A, et al. A chronic disease management programme can reduce days in hospital for patients with chronic obstructive pulmonary disease. Intern Med J. 2004;34:608-614.
24. Wakabayashi R, Motegi T, Yamada K, et al. Efficient integrated education for older patients with chronic obstructive pulmonary disease using the Lung Information Needs Questionnaire. Geriatr Gerontol Int. 2011;11:422-430.

25. Watson PB, Town GI, Holbrook N, Dwan C, Toop LJ, Drennan CJ. Evaluation of a self-management plan for chronic obstructive pulmonary disease. Eur Respir J. 1997;10:1267-1271.

26. Wood-Baker R, McGlone S, Venn A, Walters EH. Written action plans in chronic obstructive pulmonary disease increase appropriate treatment for acute exacerbations. Respirology. 2006;11:619-626.

27. Casas A, Troosters T, Garcia-Aymerich J, et al. Integrated care prevents hospitalizations for exacerbations in COPD patients. Eur Respir J. 2006; 28:123-130.

28. Coultas D, Frederick J, Barnett B, Singh G, Wludyka P. A randomized trial of two types of nurse-assisted home care for patients with COPD. Chest. 2005;128:2017-2024.

29. Effing T, Kerstjens H, van der Valk P, Zielhuis G, van der Palen J. (Cost)-effectiveness of self-treatment of exacerbations on the severity of exacerbations in patients with COPD: the COPE II study. Thorax. 2009; 64:956-962.

30. Gallefoss F, Bakke PS, Kjaersgaard PK. Quality of life assessment after patient education in a randomized controlled study on asthma and chronic obstructive pulmonary disease. Am J Respir Crit Care Med. 1999;159: 812-817.

31. McGeoch GR, Willsman KJ, Dowson CA, et al. Self-management plans in the primary care of patients with chronic obstructive pulmonary disease. Respirology. 2006;11:611-618.

32. Monninkhof E, van der Valk P, van der Palen J, van Herwaarden C, Zielhuis G. Effects of a comprehensive self-management programme in patients with chronic obstructive pulmonary disease. Eur Respir J. 2003; $22: 815-820$

33. Nguyen HQ, Donesky D, Reinke LF, et al. Internet-based dyspnea selfmanagement support for patients with chronic obstructive pulmonary disease. J Pain Symptom Manage. 2013;46:43-55.

34. Taylor SJC, Sohanpal R, Bremner SA, et al. Self-management support for moderate-to-severe chronic obstructive pulmonary disease: A pilot randomised controlled trial. Br J Gen Pract. 2012;62:e687-e695.

35. Trappenburg JC, Monninkhof EM, Bourbeau J, et al. Effect of an action plan with ongoing support by a case manager on exacerbation-related outcome in patients with COPD: a multicentre randomised controlled trial. Thorax. 2011;66:977-984.

36. Zwar NA, Hermiz O, Comino E, et al. Care of patients with a diagnosis of chronic obstructive pulmonary disease: a cluster randomised controlled trial. Med J Aust. 2012;197:394-398.

37. Majothi S, Jolly K, Heneghan NR, et al. Supported self-management for patients with COPD who have recently been discharged from hospital: a systematic review and meta-analysis. Int J Chron Obstruct Pulmon Dis. 2015;10:853-867.

38. Vanfleteren LE, Spruit MA, Groenen M, et al. Clusters of comorbidities based on validated objective measurements and systemic inflammation in patients with chronic obstructive pulmonary disease. Am J Respir Crit Care Med. 2013;187:728-735.

39. Ramachandran K, McCusker C, Connors M, Zuwallack R, Lahiri B. The influence of obesity on pulmonary rehabilitation outcomes in patients with COPD. Chron Respir Dis. 2008;5:205-209.

40. Vagaggini B, Costa F, Antonelli S, et al. Clinical predictors of the efficacy of a pulmonary rehabilitation programme in patients with COPD. Respir Med. 2009;103:1224-1230.

41. Sun X, Ioannidis JPA, Agoritsas T, et al. How to use a subgroup analysis: users' guide to the medical literature. JAMA. 2014;311:405-411.

42. Tierney JF, Vale C, Riley R, et al. Individual participant data (IPD) meta-analyses of randomised controlled trials: guidance on their use. PLoS Med. 2015;12:e1001855.

43. Hurst JR, Vestbo J, Anzueto A, et al. Susceptibility to exacerbation in chronic obstructive pulmonary disease. $N$ Engl J Med. 2010;363: $1128-1138$ 
44. Trappenburg JC, van Deventer AC, Troosters T, et al. The impact of using different symptom-based exacerbation algorithms in patients with COPD. Eur Respir J. 2011;37:1260-1268.

45. Bischoff EW, Hamd DH, Sedeno M, et al. Effects of written action plan adherence on COPD exacerbation recovery. Thorax. 2011;66:26-31.

46. Boutron I, Moher D, Altman DG, Schulz KF, Ravaud P; CONSORT Group. Extending the CONSORT statement to randomized trials of nonpharmacologic treatment: explanation and elaboration. Ann Intern Med. 2008;148:295-309.
47. Bestall JC, Paul EA, Garrod R, Garnham R, Jones PW, Wedzicha JA. Usefulness of the Medical Research Council (MRC) dyspnoea scale as a measure of disability in patients with chronic obstructive pulmonary disease. Thorax. 1999;54:581-586.

48. Linn BS, Linn MW, Gurel L. Cumulative illness rating scale. J Am Geriatr Soc. 1968;16:622-626.

\section{Publish your work in this journal}

The International Journal of COPD is an international, peer-reviewed journal of therapeutics and pharmacology focusing on concise rapid reporting of clinical studies and reviews in COPD. Special focus is given to the pathophysiological processes underlying the disease, intervention programs, patient focused education, and self management protocols.

\section{Dovepress}

This journal is indexed on PubMed Central, MedLine and CAS. The manuscript management system is completely online and includes a very quick and fair peer-review system, which is all easy to use. Visit http://www.dovepress.com/testimonials.php to read real quotes from published authors. 